JOURNAL OF SPORT \&

EXERCISE PSYCHOLOGY

\title{
Number of years of team and individual sport participation during adolescence and depressive symptoms in early adulthood
}

\begin{tabular}{|r|l|}
\hline Journal: & Journal of Sport and Exercise Psychology \\
\hline Manuscript ID & JSEP.2015-0175.R1 \\
\hline Manuscript Type: & Research Note \\
\hline Keywords: & adolescent, sport, exercise psychology \\
\hline \multicolumn{3}{|c}{} \\
\multicolumn{3}{c}{ SCHOLARONE } \\
\multicolumn{3}{c}{ Manuscripts }
\end{tabular}


Number of years of team and individual sport participation during adolescence and depressive symptoms in early adulthood 


\begin{abstract}
The purpose of this study was to examine the longitudinal and unique association between number of years of team sport and individual sport participation during adolescence and depressive symptoms during early adulthood. Adolescents $(n=844)$ reported team sport and individual sport participation in each year of secondary school for five years. Participants reported depressive symptoms using the Major Depression Inventory three years after secondary school. Multivariate linear regression was performed to model the associations of sport participation with depressive symptoms while controlling for sex, age, parent education, and baseline depressive symptoms. In the final model, adolescents who consistently participated in team sport during high school reported lower depression scores in early adulthood $(\beta=-.09, p=$ .02). Number of years of individual sport participation was not statistically significantly associated with depressive symptoms in early adulthood. Based on these findings, team sport participation may protect against depressive symptoms in early adulthood. If this finding is replicated, strategies should be implemented to encourage and maintain team sport participation during adolescence. Further research is needed to understand the mechanisms that link team sport participation to lower depression.
\end{abstract}


Approximately $30-40 \%$ of adolescent boys and girls age 12 to 19 years report moderate to high levels of depressive symptoms (Galambos, Leadbeater, \& Barker, 2004; Hankin et al., 1998), and 2 to 9\% are diagnosed with Major Depressive Disorder (MDD; Costello, Foley, \& Angold, 2006; Williams, O'Connor, Eder, \& Whitlock, 2009; Lewinsohn, Hops, Roberts, Seeley, \& Andrews, 1993). More than 50\% of adolescents diagnosed with MDD will continue to experience major depression into adulthood (Lewinsohn, Rohde, Klein, \& Seeley, 1999). Symptoms of depression can challenge overall health, well-being, and quality of life (Himelhoch et al., 2004; McIntyre et al., 2006; Judd, Schettler, \& Akiskal, 2002; Gonzalez-Tejera et al., 2005). As such, it is important to identify and test modifiable factors that may reduce depressive symptoms in youth.

Sport participation is associated with fewer or less severe depressive symptoms in youth (Boone \& Leadbeater, 2006; Gore, Farrell, \& Gordon, 2001; Sanders, Field, Diego, \& Kaplan, 2000; Ferron, Narring, Cauderay, \& Michaud, 1999). Brunet et al. (2013) found that involvement in organized sport during adolescence, but not moderate to vigorous physical activity outside of a sport context, was associated with lower depressive symptom scores in early adulthood. One explanation offered for this finding was that physical activity in the context of organized sport may foster positive mental health by providing opportunities for social interaction and connectedness. Similarly, Jewett et al. (2014) found that participation in school sport was associated with decreased depressive symptoms and stress, and higher self-rated mental health in young adulthood. These findings also highlight the potential importance of social factors in linking sport participation and mental health during adolescence and young adulthood.

The possible link between the social nature of sport participation and depression has not yet been explored longitudinally. It is possible that participation in individual sports (i.e., 
participation involving people competing against others for personal goals) is less protective than participation in team sports (i.e., involving a group of people working together toward a shared goal) due to the differences in the social nature of training and participation (Eime, Young, Harvey, Charity, \& Payne, 2013). In a cross-sectional study, Miller and Hoffman (2009) compared associations between individual sport and team sport participation and depression, and concluded that the experience of playing on a team during adolescence may contribute to the development of a prosocial athlete identity that buffers against depression. In another crosssectional study, Boone and Leadbeater (2006) showed that positive team sport involvement (i.e., involvement that led to skill development, and which had warm positive coaching and fostered feelings of social support and belonging), mediated the risk of depressive symptoms in male and female adolescents. In a longitudinal study spanning two years, Vella et al. (2014) reported that children aged 8 to 10 years who participated in team sports had greater health-related quality of life (a construct that includes assessment of psychological health) over time compared to children who only participated in individual sports. Possible explanations included that team sports enhance perceived social acceptance (Boone \& Leadbeater, 2006), teamwork and accountability to others (MacDonald, Cote, Eys, \& Deakin, 2012), collective goal-striving (Larson, 2011), and emotional regulation (Vella, Oades, \& Crowe, 2013). Team sport may also be an environment conducive to social contagion effects (Scarapicchia et al., 2013), and may lead to more intense and more frequent physical activity, which may improve mental health outcomes through neurobiological influences such as monoamine availability and increased neurotrophic factors (aan het Rot, Collins, \& Fitterling, 2009). Although youth can participate in both team and individual sport, and despite the possible differences in the social and training 
contexts of team and individual sport participation, there has been limited attention in exploring the possible differences in their association to mental health.

Although the notion that sport participation may protect against depression has been examined, there has been little attention directed to the unique benefits offered by team and individual sports participation. Evaluating which type of sport participation is associated with depression may inform sport programing that is concerned with promoting mental health as well as mental health interventions. In the current study, the longitudinal association between participation in team and individual sports in adolescence and depressive symptoms in early adulthood was examined. We hypothesized that number of years of involvement in team sport would be associated with fewer depressive symptoms during early adulthood, whereas no such relationship would emerge for individual sport participation.

\section{Method}

Data for the study were drawn from the Nicotine Dependence In Teens (NDIT) study, which is a prospective cohort study of 1294 students ( $52 \%$ female) who were enrolled in one of 10 purposefully recruited high schools in Montreal, QC. Beginning in 1999, the first 20 survey cycles comprised self-report questionnaires administered at schools every three months during the 10-month school year for the five years of secondary school. Additional data were collected three years after graduation from high school (2008-09; survey cycle 21) in mailed self-report questionnaires completed by 880 participants. Approval from appropriate ethics committees was obtained for the study protocol. Further details on the study design have been published elsewhere (O’Loughlin et al., 2014).

A total of 844 males and females who provided complete data were included in the analytic sample. Participants (54\% female) were on average 20.4 (standard deviation $[\mathrm{SD}]=0.7)$ 
years of age at survey cycle 21. Mean depressive symptoms at baseline on a scale ranging from 1

to 4 were $2.1(\mathrm{SD}=0.6)$. There were no significant differences in baseline age, sex, parent

education, team or individual sport participation, or depressive symptoms between participants

retained and not retained in the analysis.

\section{Measures}

Involvement in team and individual sport. Involvement in team sport and individual sport was measured in survey cycles 1 through 20 by asking participants if they belonged to any of 12 sport teams at school (basketball, soccer, football, track and field, rugby, wrestling, swimming, softball, cross-country ski, volleyball, gymnastics, and hockey) and 11 sport teams outside of school (basketball, soccer, football, swimming, baseball, volleyball, hockey, ballet/dance, aerobic classes, ski lessons, and judo/karate). The list of sports for both individual and team participation was based on common opportunities in Canadian schools and recreational and community programs at time of the baseline assessment period. Participants were also offered the chance of providing responses for "other". Response options for each sport were 'yes' or 'no'. Sports were categorized into team (football, basketball, volleyball, soccer, hockey, softball, baseball, and rugby) or individual (swim, track, gymnastics, dance, cross-country ski, wrestling, and judo) categories based on the primary competition context. As such, coactive sports were included in the individual sport category because participation requires little to no group interaction and coordination for the achievement of goals. Sports listed as "other" were also categorized as team or individual (e.g., ringette). To account for seasonal variation in opportunities for involvement and participation, responses across a single school year (September to June) were collapsed into a dichotomous variable (yes or no) for each of the five years of secondary school. As such, both team sport and individual sport participation scores 
ranged from 0 to 5 (no involvement to 5 years of involvement). For descriptive purposes only, participants were also coded as involved in no sport, or team sport, individual sport, or both team and individual sport across the five years of adolescence.

Depressive Symptoms. Depressive symptoms were assessed in survey cycle 21 (three years after secondary school) using the Major Depression Inventory (MDI; Bech, Rasmussen, Olsen, Noerholm, \& Abildgaard, 2001). Participants reported the frequency of experiencing 10 depressive symptoms in the past two weeks using a six-point Likert-type scale ranging from 0 ("at no time") to 5 ("all the time"). The scores for each symptom were summed to create the MDI score that ranged from 0 to 50 . For items 8 and 10 of the MDI, which each had two possible choices (a and b), only the highest of the two scores was used as per recommended scoring procedures for the MDI (Bech et al., 2001). Scores on the MDI are valid and reliable in adults (Bech et al., 2001). In the current study, the Cronbach's alpha was 0.9.

Covariates. Potential confounders of the associations between team and individual sport participation and depressive symptoms assessed at survey cycle 21 included sex (male $=1$ or female=2), age, baseline depression symptoms, and parent education. Baseline depression symptoms were measured using a six-item depressive symptoms scale at survey cycle 1 (Kandel \& Davies, 1982), for which scores have been shown to be valid and reliable (Brunet, Sabiston, Chaiton, Low, Contreras, Barnett, \& O’Loughlin, 2014). Participants indicated the frequency with which they experienced six symptoms related to depression in the past 3 months on a 4point Likert-type scale ranging from 1 (never) to 4 (often). Responses were summed and averaged to create the baseline depression symptom score. Parental education status, used as a proxy for socioeconomic status, was dichotomized as "no university education" (score $=0)$ or "one or both parents have some university education" (score $=1$ ) using data reported by parents 
themselves around the same time as participants completed survey cycle 21 . If data were missing from parents, data reported by participants in survey cycles 13 or 17 were used.

\section{Data analysis}

Data analyses were performed in SPSS V20 (IBM Corp., Armonk, NY). Descriptive statistics (means, standard deviations (SD), Pearson correlation coefficients) were computed in preliminary analyses. Hierarchical linear regression was used to model the associations of number of years of participation in team sport and individual sport during adolescence with depressive symptoms in early adulthood. Covariates (sex, age, baseline depressive symptoms, and parent education) were entered on step 1, and team and individual sport participation variables were entered on step 2. Cohen's $f^{2}$ (Cohen, 1988) was calculated as $f^{2}=R^{2} \div\left(1-R^{2}\right)$ to provide effect size coefficients. In sensitivity analysis, separate models tested if team or individual sport participation were independent predictors of depressive symptoms. To explore the unique effects of team and individual sport experiences, we also tested a third model by excluding participants who competed in both team and individual sports at any time during adolescence.

\section{Results}

Participants reported an average of $2.4(\mathrm{SD}=2.0)$ years in team sport and $1.6(\mathrm{SD}=1.7)$ years in individual sport. In the sample, $12.6 \%$ of the participants reported no team or individual sport participation; and 52\% of the sample reported participating in both team and individual sport in one or more data collection cycles during adolescence. The mean MDI score for depressive symptoms at survey cycle 21 was $9.8(\mathrm{SD}=7.8)$. Mean MDI scores for participants involved in no sports $(n=108)$, individual sports only $(n=103)$, team sports only $(n=200)$, or 
both team and individual sports $(n=449)$ at any point during adolescence were $11.08(\mathrm{SD}=$ 9.36), $11.14(\mathrm{SD}=8.53), 8.73(7.27)$, and $9.64(7.51)$ respectively.

Years of involvement in team sport participation during adolescence was positively correlated with years of participation in individual sport during the same time period $(r=.22, p=$ $.01)$, and negatively correlated with depressive symptoms in early adulthood $(r=-.12, p=.01)$. The number of years of individual sport participation was positively, but not statistically significantly, correlated with depressive symptoms $(r=.04, p=.30)$.

The regression model was statistically significant, $F(6,838)=15.71, p<.001, R^{2}=0.12$, Cohen's $f=0.14$ (Table 1). After controlling for sex, age, baseline depressive symptoms, and parent education, years of team sport participation during adolescence was negatively associated with depressive symptoms in early adulthood. The number of years of individual sport participation was not significantly associated with depressive symptoms in early adulthood. The findings were consistent when the number of years of team (Model $R^{2}=0.11$, team sport coefficient $\beta=-0.09, \mathrm{p}=.03$ ) and individual (Model $R^{2}=0.09$, individual sport coefficient $\beta=$ $0.02, p=.94)$ sport participation were tested in separate models, as well as in the subset of participants who reported experiences in team or individual sport exclusively. Among the 481 participants reporting individual $(n=164)$ or team $(n=317)$ sport only throughout adolescence, the number of years of team sport involvement was a significant predictor of depressive symptoms $(\beta=-0.11, p=.03)$ after controlling for the covariates whereas number of years of involvement in individual sport was not significant $(\beta=0.04, p=0.39)$.

\section{Discussion}

The association between team sport and individual sport participation during five years of high school and depressive symptoms three years later in early adulthood was examined in the 
current study. Based on these findings, number of years in team sport participation, but not in individual sport participation, is longitudinally associated with depressive symptoms after controlling for socio-demographic variables. Although the effects in the model predicting depressive symptoms were small and should be replicated, prevention efforts aimed at reducing the prevalence and burden of depressive symptoms could be directed at promoting consistent team sport involvement throughout adolescence.

Consistent with the hypothesis, adolescents who participated in more years of team sports during secondary school reported lower depressive symptoms in early adulthood. Participation in sports in general has previously been associated with fewer or less severe depressive symptoms in cross-sectional and longitudinal studies (Boone \& Leadbeater, 2006; Brunet et al., 2013; Gore et al., 2001; Sanders et al., 2000; Ferron et al., 1999). Based on the current study results, number of years participating in team sport may protect against depressive symptoms. These findings were robust in sensitivity analyses when team and individual sport participation were tested in separate models and when participants playing both team and individual sport were excluded. It was also evident in the mean values of depression symptoms, whereby participants involved in team sport reported the lowest MDI scores compared to peers involved in both team and individual sport, individual sport only, or no sport. The team sport climate offers a possible explanation for this finding. Based on a review of the psychological and social benefits of sport participation, Eime and colleagues (2013) suggest that due to the social nature of teams, team sport participation contributes more strongly to positive psychological outcomes such as lower depressive symptoms than individual sport participation. In addition, team sports may reduce depressive symptoms indirectly by fostering a positive athletic identity (Miller \& Hoffman, 2009), building self-esteem (Pederson \& Siedman, 2004), and a sense of social acceptance 
(Boone \& Leadbeater, 2006). Further, a wide range of team sports meets the description of vigorous intensity exercise, which increases monoamines in the brain that help stabilize mood (e.g., serotonin and dopamine), immune function, and quality of life factors that are linked to mental health (aan het Rot, Collins, \& Fitterling, 2009; Sabiston, Castonguay, Low, Barnett, Mathieu, O’Loughlin, \& Lambert, 2010; Velle et al., 2014). Possible mechanisms linking team sport participation and depressive symptoms need to be tested in future research.

In the current study, individual sport was not statistically significantly associated with depressive symptoms and the beta coefficient was small suggesting that there is little association between individual sport participation and depressive symptoms. The number of years in individual sport participation may not be as protective against depression as number of years of team sport experiences due to lack of social connections and cohesion that may limit improvements in self-concept, self-esteem, and foster feelings of social isolation (Barber, Eccles, \& Stone, 2001; Eime et al., 2013; Marsh, 1993; Pedersen \& Siedman, 2004). In addition, many individual sports are judged by others or emphasize physical appearance along with performance (Parsons \& Betz, 2001). As such, the differences in associations of team sport and individual sport participation with mental health may relate to sport-specific and contextual mechanisms. In summary, and consistent with the results of the current study, researchers have identified weaker relationships between individual sport participation and psychological, social, and quality of life outcomes (Eime et al., 2013; Vella et al., 2014). Further research efforts are needed to understand the mechanisms that help to explain to association.

Limitations of this study include use of a convenience sample which may limit generalizability of the results. Data were collected using self-report measures that may be subject to misclassification bias. The classification of sports as team or individual may not capture social 
contextual differences in participation. There may also be a biased sample such that youth who experienced depressive symptoms early in adolescence may have opted out of team sport. Selection bias due to loss to follow-up is also possible, although participants in the sample retained for the analyses were not significantly different at baseline from those who dropped out on age, sex, parent education, or team and individual sport participation, or depressive symptoms. Lastly, although we controlled for several potential confounders, unmeasured variables associated with sport participation and depressive symptoms, such as self-esteem, social connectedness and acceptance, and body image may explain the observed association. There may also be personal and contextual factors associated with the selection of individual sport participation over team sports that are associated with depressive symptoms. Despite these limitations, this study has strengths including a large sample size, low attrition rate from high school to young adulthood, use of a longitudinal study design spanning adolescence and early adulthood, and valid and reliable measures used to assess depressive symptoms.

To conclude, this study is the first to show that the number of years of team sport participation, but not years of involvement in individual sport, during adolescence is associated with depressive symptoms in early adulthood. Although replication of these findings is necessary, they provide support that widespread strategies need to be implemented to help more adolescents participate, and stay involved, in team sports to protect against depressive symptoms in early adulthood. Finally, while we can merely speculate on the social aspects inherent in team sport participation that may be driving these associations, future research examining the contribution of such social aspects to the benefits of team sport participation is needed. 


\section{References}

aan het Rot, M., Collins, K., Fitterling, H. L. (2009). Physical activity and depression. Mount Sinai Journal of Medicine, 76, 204-14. doi: 10.1002/msj.20094

Barber, B., Eccles, J., Stone, M. (2001). Whatever happened to the jock, the brain, and the princess? Young adult pathways linked to adolescent activity involvement and social identity. Journal of Adolescent Research, 16, 429-455. doi: 10.1177/0743558401165002

Bech, P., Rasmussen, N. A., Olsen, L. R., Noerholm, V., Abildgaard, W. (2001). The sensitivity and specificity of the Major Depression Inventory, using the Present State Examination as the index of diagnostic validity. Journal of Affective Disorders, 66, 159-64. http://dx.doi.org/10.1016/S0165-0327(00)00309-8

Boone, E., Leadbeater, B. (2006). Game on: Diminishing risks for depressive symptoms in early adolescence through positive involvement in team sports. Journal of Research on Adolescence, 16, 79-90. doi: 10.1111/j.1532-7795.2006.00122

Brunet, J., Sabiston, C. M., Chaiton, M., Barnett, T.A., O’Loughlin, E., Low, N. C. P., O'Loughlin. J. (2013). The association between past and current physical activity and depressive symptoms in young adults: a 10-year prospective study. Annals of Epidemiology, 23, 25-30. doi: 10.1016/j.annepidem.2012.10.006

Brunet, J., Sabiston, C. M., Chaiton, M., Low, N. C. P., Contreras, G., Barnett, T.A., \& O’Loughlin. J. (2014). Measurement invariance of the depressive symptoms scale during adolescence. BMC Psychiatry, 14:95. doi: 10.1186/1471-244X-14-95

\section{Cohen J. E. (1988). Statistical Power Analysis for the Behavioral Sciences. Hillsdale, NJ:} Lawrence Erlbaum.

Costello, E. J., Foley, D. L., Angold, A. (2006). 10-year research update review: The epidemiology of child and adolescent psychiatric disorders: II. Developmental epidemiology. Journal of the American Academy of Child and Adolescent Psychiatry, 45, 8-25. http://dx.doi.org/10.1097/01.chi.0000184929.41423.c0

Eime, R. M., Young, J. A., Harvey, J. T., Charity, M. J., Payne, W. R. (2013). A systematic review of the psychological and social benefits of participation in sport for children and adolescents: Informing development of a conceptual model of health through sport. International Journal of Behavioral Nutrition and Physical Activity, 10, 98. doi:10.1186/1479-5868-10-98

Ferron, C., Narring, F., Cauderay, M., Michaud, P. (1999). Sport activity in adolescence: Associations with health perceptions and experimental behaviors. Health Education Research, 14, 225-233. doi: 10.1093/her/14.2.225 
Galambos, N. L., Leadbeater, B. J., Barker, E. T. (2004). Gender differences and risk factors for depression in adolescence: A 4-year longitudinal study. International Journal of Behavioral Development, 28, 16-25. doi: 10.1080/01650250344000235

Gonzalez-Tejera, G., Canino, G., Ramirez, R., Chavez, L., Shrout, P., Bird, H., Bauermeister, J.(2005). Examining minor and major depression in adolescents. Journal of Child Psychology and Psychiatry, 46, 888-99. doi: 10.1111/j.1469-7610.2005.00370

Gore, S., Farrell, F., Gordon, J. (2001). Sports involvement as protection against depressed mood. Journal of Adolescent Research, 11, 119-130. doi: 10.1111/1532-7795.00006

Hankin, B., Abramson, L., Moffitt, T., Silva, P., McGee, R., Angell, K. (1998). Development of depression from preadolescence to young adulthood: Emerging gender differences in a 10-year longitudinal study. Journal of Abnormal Psychology, 107, 128-140. doi: 10.1037/0021-843X.107.1.128

Himelhoch, S., Lehman, A., Kreyenbuhl, J., Daumit, G., Brown, C., Dixon, L. (2004). Prevalence of chronic obstructive pulmonary disease among those with serious mental illness. American Journal of Psychiatry, 161, 2317-9. http://dx.doi.org/10.1176/appi.ajp.161.12.2317

Jewett, R., Sabiston, C. M., Brunet, J., O’Loughlin, E. K., Scarapicchia, T., O’Loughlin, J. (2014). School sport participation during adolescence and mental health in early adulthood. Journal of Adolescent Health, 55, 640-644. http://dx.doi.org/10.1016/j.jadohealth.2014.04.018

Judd, L. L., Schettler, P. J., Akiskal, H. S. (2002). The prevalence, clinical relevance, and public health significance of subthreshold depressions. Psychiatric Clinics of North America, 25, 685-98. http://dx.doi.org/10.1016/S0193-953X(02)00026-6

Kandel, D. B., \& Davies, M. (1982). Epidemiology of depressive mood in adolescents: An empirical study. Archives of Generaly Psychiatry, 39, 1205-12.

doi:10.1001/archpsyc.1982.04290100065011

Lewinsohn, P. M., Hops, H., Roberts, R. E., Seeley, J. R., Andrews, J. A. (1993). Adolescent psychopathology: I. Prevalence and incidence of depression and other DSMIII-R disorders in high school students. Journal of Abnormal Psychology, 102, 133-44. http://dx.doi.org/10.1037/0021-843X.102.1.133

Lewinsohn, P. M., Rohde, P., Klein, D. N., Seeley, J. R. (1999) Natural course of adolescent major depressive disorder: I. Continuity into young adulthood. Journal of the American Academy of Child and Adolescent Psychiatry, 38, 56-63.

doi: 10.1176/appi.ajp.157.10.1584

Marsh, H. (1993). The effects of participation in sport during the last two years of high school. Sociology of Sport Journal, 10, 18-43. Retrieved from http://journals.humankinetics.com/ssj 
MacDonald, D. J., Côté, J., Eyes, M., \& Deakin, J. (2012). Psychometric properties of the youth experience survey with young athletes. Psychology of Sport and Exercise, 13, 332-340. Doi:10.1016/j.psychsport.2011.09.001.

McIntyre, R. S., Konarski, J. Z., Soczynska, J. K., Wilkins, K., Panjwani, G., Bouffard, B., ... Kennedy, S. H. (2006). Medical comorbidity in bipolar disorder: Implications for functional outcomes and health service utilization. Psychiatric Services, 57, 1140-4. Retrieved from http://ps.psychiatryonline.org/

Miller, K., Hoffman, J. (2009). Mental well-being and sport-related identities in college students. Sociology of Sport Journal, 26, 335-356. Retrieved from http://journals.humankinetics.com/ssj

O’Loughlin, J., Dugas, E. N., Brunet, J., DiFranza, J., Engert, J. C., Gervais, A., et al. (2014). Cohort profile: The Nicotine Dependence in Teens (NDIT) Study. International Journal of Epidemiology. doi: 10.1093/ije/dyu135

Parsons, E. M., \& Betz, N. E. (2001). The relationship of participation in sports and physical activity to body objectification, instrumentality, and locus of control among young women. Psychology of Women Quarterly, 25, 209-222. doi: 10.1111/1471-6402.00022

Pedersen, S., Siedman, E. (2004). Team sports achievement and self-esteem development among urban adolescent girls. Psychology of Women Quarterly, 28, 412-422.

doi: $10.1111 /$ j.1471-6402.2004.00158

Sabiston, C. M., Castonguay, A., Low, N., Barnett, T., Mathieu, M., O’Loughlin, J., \& Lambert, M. (2010). Vigorous physical activity and low-grade systemic inflammation in adolescent boys and girls. International Journal of Pediatric Obesity, 5, 509-515. doi:

$10.3109 / 17477160903572019$

Sanders, C., Field, T., Diego, M., Kaplan, M. (2000). Moderate involvement in sports is related to lower depression levels among adolescents. Adoelscence, 35, 793-798. doi: $10.1177 / 216770261245048$

Vella, S. A., Cliff, D. P., Magee, C. A., \& Okely, A. D. (2014). Sports participation and parentreported health-related quality of life in children: Longitudinal association. Journal of Pediatrics, 164, 1469-1474. doi:10.1016/j.jpeds.2014.01.071

Vella, S. A., Oades, L. G. \& Crowe, T. P. (2013). A pilot test of transformational leadership training for sports coaches: Impact on the developmental experiences of adolescent athletes. International Journal of Sports Science and Coaching, 8 (3), 513-530. doi: http://dx.doi.org/10.1260/1747-9541.8.3.513

Williams, S. B., O’Connor, E. A., Eder, M., Whitlock, E. P. (2009). Screening for child and adolescent depression in primary care settings: A systematic evidence review for the US Preventive Services Task Force. Pediatrics, 123, 716-35. doi: 10.1542/peds.2008-2415 
Table 1.

Mean number of years of team and individual sport participation during adolescence as predictors of mean depressive symptoms scores during young adulthood $(n=844)$.

\begin{tabular}{|r|c|c|c|}
\hline & $\mathrm{b}(\mathrm{SE})$ & $\beta$ & $\mathrm{R}^{2}$ \\
\hline Step 1 & & & 0.09 \\
\hline Sex & $3.15(0.55)$ & $0.20^{*}$ & \\
\hline Age & $-0.18(0.38)$ & -0.02 & \\
\hline Baseline depression & $2.85(0.45)$ & $0.22^{*}$ & \\
\hline Parent Education & $-0.27(0.24)$ & -0.04 & \\
\hline & & & 0.12 \\
\hline Sex & $2.85(0.58)$ & $0.18^{*}$ & \\
\hline Age & $-0.10(0.38)$ & -0.01 & \\
\hline Baseline depression & $2.78(0.46)$ & $0.21^{*}$ & \\
\hline Parent education & $-0.43(0.25)$ & -0.06 & \\
\hline Years of team sport & $-0.33(0.14)$ & $-0.09^{*}$ & \\
\hline Years of individual sport & $0.05(0.17)$ & 0.01 & \\
participation & 0.02 & \\
\hline
\end{tabular}

*Statistically significant at $p<0.05$

${ }^{1}$ Sex was coded as male $=1$ and female $=2$; parent education was coded as less than university education $=0$ and university education $=1$ 